\begin{tabular}{ccc}
\hline & International Journal of Engineering \& Technology, $7(2.32)(2018) 405-407$ \\
SPC & International Journal of Engineering \& Technology \\
Website: www.sciencepubco.com/index.php/IJET & Research paper \\
\hline
\end{tabular}

\title{
Operational Multi-Modal Distance Metric Learning to Image Reclamation
}

\author{
L Lavanya $^{1}$, Chebrolu Ujwala Pavani ${ }^{2}$, Gadchanda Vineeth $^{3}$, Borada Lavanya ${ }^{4}$ \\ ${ }^{1,2,3,4}$ Dept of CSE, KL E F,Vaddeswaram, India \\ *Corresponding author E-mail: lavanyareddy@kluniversity.in
}

\begin{abstract}
Distance learning is an eminent technique that improves the search for images based on content. Although widely studied, most DML approaches generally recognize a modalization training framework that teaches a metric distance or a combination of distances in which several types of characteristics are simply interconnected. DML methods of that type suffer some critical limitations (a) Some feature types can significantly overwhelm others with the DML assignment, due to different attributes, and (b) the distance learning standard in the combined metric properties can be consumed using the feature attribute approach combined. In this article we refer to these the restrictions are reviewed online- multimodal distance metric training scheme (OMDML), which explores a dual duplication online learning scheme. (c) learn to optimize the distance metric in each owner space separately; and (d) learn find the optimal combination of different types of characteristics. To overestimate the cost of DML in sophisticated areas, we offer a low level OMDML algorithm that not only reduces estimated costs, but also guarantees high accuracy. We are here carried out exhaustive experiments to estimate the performance of the algorithms proposed for the restoration of multimedia images.
\end{abstract}

Keywords:Multi-Modal Distance, Image Reclamation.

\section{Introduction}

One of the main research tasks of multimedia recovery is to calculate the similarity of two objects in content-based multimedia recovery tasks with the function / effective distance metric. In the last decades, multimedia researchers have made great efforts in different designs low level functions and different distance measures. The problem of finding a decent separation/work meter remains an open test for multimedia content recovery problems. In recent years, it is a promising direction This challenge will focus on the study of distance learning through the application of machine learning methods, the optimization of training data or remote sensors, such as historical records for user compliance in image content recovery systems. Although some are DML algorithms in the literature have suggested that most existing DML methods belong to a single-mode DML as they take a metric specification of type or a combination of distance, combining different characteristics In the real world, such approaches to practice may experience the ill effects of some pragmatic constraints. The types of operational types can significantly overwhelm others in the DML task by loosening the capabilities of all functions and the intelligent concentration of concentration can result in packets large dimensional plane, which transforms the following computationally intensive DML activity. To overcome the aforementioned limitations, this document examines the range of new distance learning algorithms multimedia data standards or multiple types of functions with an effective and extensive online learning scheme. Unlike the concentration approach mentioned above, the two main ideas are: Learn how to optimize individual distance measurements for every individual mode and learn to find the optimal combination of multiple distance standards in multiple modes. In addition, OMDML uses online teaching methods for a large-scale, high-efficiency, large-scale educational activity. To reduce future billing costs, we also offer a low quality online DML algorithm (LOMDML) that avoids.

\section{Related Work}

\subsection{Appearance-Based Methods}

There are many varieties that are offered, for example, with global or regional accents and excellent reviews. Many strategies have coordinated some of the most important events that take into account the final objective of important events. The spatial data on the design of these important projects are also equally important. The task of false images is the symmetric collection of local features that have been found in parts of visible vision and symmetry standards. The maximum strategy focuses on the plan, while others are different from the clarity of the promising elements was advantageous in the description of each specific person, and the mining industry of significant importance could have been implemented without any doubt.

\subsection{Learning-Based Methods}

The methods of teaching special elements are used as a validation as an assistance vector machine and are generally promoted. These strategies and adults bring the problem to bidirectional or multiparty classification. In any case, Prosser reaffirms in depth his position as a posture. It has developed a first class SVM classifier and has the ability to achieve a direct weight gain The difference between the tests. The relative distance comparison (RDC) considers the combined effect of several important events using a 
single time that includes quantitative visualization. RDC can be seen as a special fluctuation of the mathematical learning calculation for characteristics. The metric training calculations are taught in the month, which is all more capable of quantitative It includes contrasts. In addition, the trained mathematical method is under the metric training system. Over time, the early methodology of moderate metric training has led to rapid lighting strategies for duplication investigations. Consequently, these strategies require the preparation of tests for typical names and large-scale separation. Proposed approach to work with the grouping metric and incremental separation, the iterative adaptation of the online broadband pool to calculate the altitude information: He used Adaptive Metric Learning to isolate cluster clusters by removing the edges of the nerve joints. The main contrast between our technique and our predecessor is that they concentrate on raising the nature of the information gang, while our chips move away from the method of gradual division.

\section{Online Learning}

A large-scale study of distance learning in communities of machine learning and rehabilitation of multimedia is carried out. The essential thought is to take in the ideal criteria that limits the separation between comparable/related pictures and boost the separation between various/boundless pictures. Existing DML Studies can be ordered by various classes as per distinctive standards and rules of learning. For suppose, the interaction of several messenger disorders, the DML technique is divided mainly into two groups. Global controlled approaches: Improved in a global metric field, for example, all constraints will be performed simultaneously. Localized approaches. For local measurements, for example, local restrictions must be given to neighboring information.

Later, according to the different types of learning data, DMLs for machine learning are generally used for measurement of direct measurement, while multimedia LMD studies usually use the measurement of secondary data to obtain the following two shapes. Limitations in pairs. contains divided pairs, that is, $p$ is connected / similar. T means the depth of the total density of the triangle. When only explicit class labels are provided, secondary information can also be constructed only when the relationships between the same object and the relationships of different classes do not match. In our work we focus Triple obstacles Finally, the DML studies existing in the teaching methodology are mainly used in group teaching methods that generally involve the whole collection of training data before instruction and zero learning, with the exception of recent studies online Training Methods: All these activities are mainly related to the DML, that vary from the different DMLs. We additionally take note of that our work is altogether different from the current DML analysis, That is related to uniform problems of arrangement, teaching class data with clear class labels, which compare our method. Keep in mind that our work differs from the study of other multimodal research focused on a wide range of search engines where multidimensional learning is formulated in a comprehensive study to optimize Specific functionality based on the loss of specific facial expressions in search engines unauthorized information. At last, note that our work differs from existing distance education thinks that teach farreaching non-secondary functions using nuclear teaching methods. Unlike the linear distance meter training methods, the core methods can be it generally reaches some of the scenarios of greater learning precision, but decreases in the growth of the limitation curve for large-scale programs, that is, the cost of enrollment increases markedly when training samples are growing. Consequently, our empirical study focuses on the direct comparison of family linear methods.

\subsection{Algorithm for Incremental Learning Process}

Input:

Nodes set N;

Count a.

Output:

Updated distance metric M.

1. Remove d "noise" nodes;

2. $\mathrm{a}=\mathrm{a}+\mathrm{d}$

3. if $\mathrm{a} \geq\|\mathrm{N}\| / \alpha$

4. $\operatorname{Ssim}=\operatorname{Sdis}=\{\}$;

5. for Node $i \in \mathrm{N}$ do

6. Search the nearest neighbor points Ni $\mathrm{n}$ for $\mathrm{i}$;

7. $\operatorname{Sisim}=\{(x i a, x i b) \mid a=1, \ldots, n, b=\operatorname{rand}(n), b=a\}$

8. Randomly select a node $j$ from $N, j \in N \backslash\{i\}$;

9. $\quad$ Sidis $=\{(x i a, x j b) \mid a=1, \ldots, n, b=\operatorname{rand}(n), i=j\}$;

10. Ssim $=$ SsimSisim, Sdis $=$ SdisSidis

11. end for

12. $\mathrm{M}=\mathrm{KISSME}(\operatorname{SsimSdisS} 0)$;

13. $\mathrm{a}=0$;

14. end

\subsection{Algorithm Explanation}

The only way to solve the problem is to optimize it. Approach to package knowledge This, yet, is not a respectable solution, mainly for two main reasons. The critical aspect of this batch training solution is that it is spent on dual training, that is, when there is a new learning model, the whole model must be completely updated from scratch so that the implemented programs are measurable. In addition to the report solution. It can be same exclusive and have a large volume of exercise data. Face to face challenges, we display a web based learning calculation to address the issue of multicultural reserve learning. Multiple meter online. is an important challenge for learning problems, to develop an effective and extensive training plan that can optimize the remote measurement in each individual model and at the same time optimize the combined weights of different models. For this purpose, we suggest investigating Remote distance metric learning algorithm, that is, the measurement of individual distance measurements of OASIS and PD and the known Benefit algorithm for optimal combined weights. We are discussing each of these two studies the following tasks. Let us examine the space-model model - step. To find the optimal criteria for individual models, after the same ideas, we can formulate the problem of optimizing the learning method online

\section{Appraisal on the Standard Datasets}

In this section, we create four collections of data, titles and thumbnails with the appropriate standard data collection. When you first select 10 categories of objects, randomly select 50 copies per class. We recognize the global characteristics mentioned above as multimedia modes. Create Three limitations of the online learning approaches, we create all the positive pairs of fragments of episodes of the same class and we randomly select an image of another class for each positive pair. In total, around 10 pounds per data. Given table describes the results of the evaluation in smaller data collections of which we can describe the following comments: First, the two types of fusion strategy i.e , before stratification and deferred node, are usually better than one of the best onedimensional approaches. This is mainly due to a combination of several different attributes that can be studied better the potential of all the characteristics that validate the proposed technique. Second, the only combined algorithm in some cases failed to improve the best one-dimensional approach. and LMNN-U in Caltech101(S). This implies that unified communication is not optimal, but merges original types of attributes. Therefore, it is very important, When training cars, they reveal the effective characteristics that give them more weight. Third, the algorithms offered by 
OMDML and LOMDML in all the coherent algorithms beat alternate algorithms. After all, it is fascinating to note that the proposed low level algorithm is not it just enhances the proficiency and measures of OMDML, yet in addition adds to the exactness of the inquiry. This is likely due to the fact that learning standards in the internal area can avoid over population and clash problems. Following chart indicates the time value of the current data processor. We see that the start time of LOMDML supposes the Coefficient of comparative speed of 10 OASIS and the increase of the productivity will increment when it increases the data collection or the volume of data. Conversely, OMDML is too high because the PSD is a PSD program that can be a dense matrix after each repetition. The possible solution to this problem is that we can do it.

\section{Experimental Results}

Evaluation of the mAP Performance

\begin{tabular}{lcccc}
\hline Alg. & COREL & Caltech101 & Indoor & ImageCLEF \\
\hline Eucl-B & 0.1877 & 0.2187 & 0.0469 & 0.5523 \\
RCA-B & 0.2305 & 0.2837 & 0.0499 & 0.6010 \\
OASIS-B & 0.1958 & 0.3025 & 0.0522 & 0.6723 \\
Eucl-C & 0.2628 & 0.2259 & 0.0559 & 0.5752 \\
RCA-C & 0.2714 & 0.2473 & 0.0604 & 0.6272 \\
OASIS-C & 0.3202 & 0.3660 & 0.0726 & 0.7394 \\
Eucl-U & 0.2628 & 0.2259 & 0.0559 & 0.5752 \\
RCA-U & 0.2992 & 0.2413 & 0.0565 & 0.6161 \\
OASIS-U & 0.3594 & 0.3243 & 0.0705 & 0.6891 \\
LOMDML & $\mathbf{0 . 4 1 3 7}$ & $\mathbf{0 . 4 1 2 8}$ & $\mathbf{0 . 0 8 0 4}$ & $\mathbf{0 . 8 1 5 5}$ \\
\hline
\end{tabular}

From the above dataset values we are generating the graph

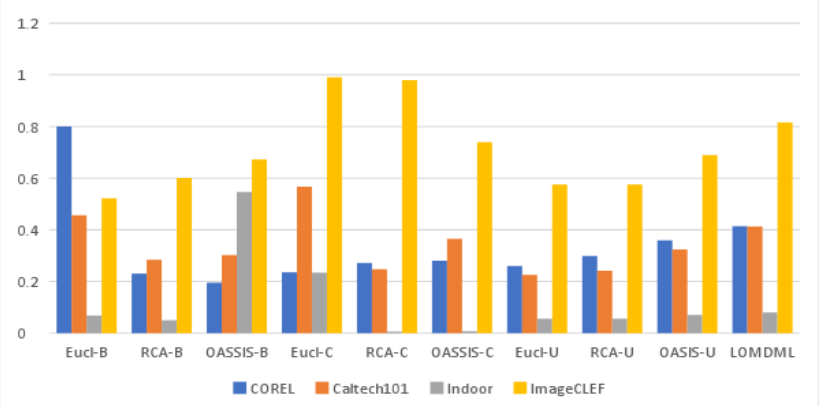

Fig.1. Values of current data processor

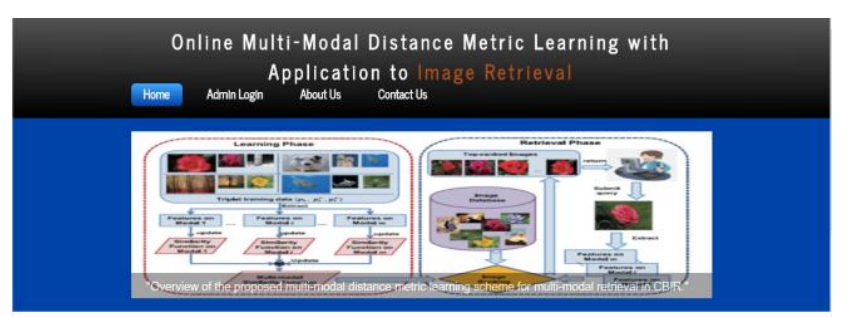

Online Mutt-Modal Distance Metric Leaming with Application to Image

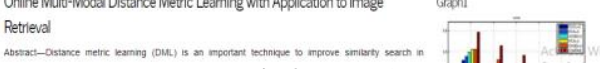

Fig 2: Sample Screen

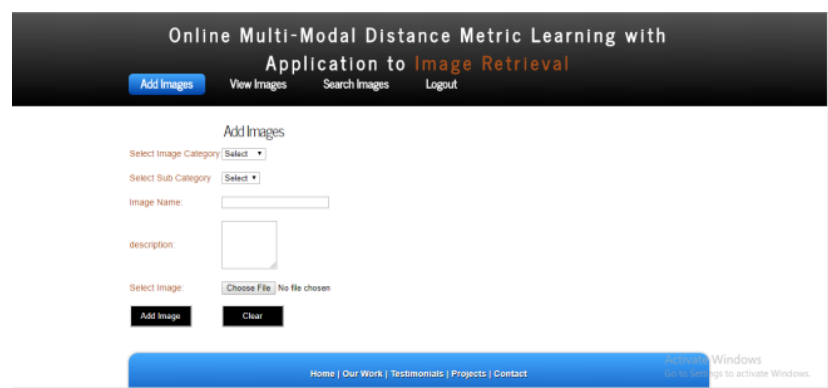

Fig 3:. Filling Form

\section{Conclusion}

This paper examined the new generation multimodal online metric training algorithms, using CIR tasks that use multiple types. We support some of the main DML comes close and introduces the online DML multimedia method. At the same time, learn the optimal distance, as well as in each individual space and the optimal combination different sizes and sizes. In addition, we have introduced a low density multimedia DML algorithm that not only does not work efficiently and extensively, but also works with the modern performance of competitive algorithms in this evaluation. Future work can extend our systems for different sorts of multi media information examination, with the exception of images.

\section{References}

[1] M. S. Lew, N. Sebe, C. Djeraba, and R. Jain, "Contentbasedmultimedia information retrieval: State of the art and challenges," ACMTrans.Multimedia Comput., Commun. Appl., vol. 2, no. 1, pp. 1-19, 2006

[2] Y.Jing and S. Baluja, "Visualrank: Applying pagerank to Largescale image search," IEEE Trans. Pattern Anal. Mach. Intell., vol. 30, no. 11 , pp. 1877-1890, Nov. 2008.

[3] D.GrangierandS.Bengio,"A discriminative Kernel-based approach to rank images from text queries," IEEE Trans. Pattern Anal. Mach. Intell., vol. 30, no. 8, pp. 1371-1384, Aug. 2008.S. C. Hoi, W. Liu, and

[4] S.-F. Chang, "Semi-supervised distance metric learning for collaborative image retrieval," in Proc. IEEE Conf. Comput. Vis. Pattern Recog., Jun. 2008, pp. 1-7.

[5] J.Sivic, B.C. Russell, A. A. Efros, A. Zisserman, and W. T. Freeman, "Discovering objects and their location in images," in Proc. IEEE Conf. Compute. Vis. Pattern Recog., 2005, pp. 370-377.

[6] A. Globerson and S. Roweis, "Metric learning by collapsing classes," in Proc. Adv. Neural Inf. Process. Syst., 2005, pp. 451-458.

[7] L. Yang, R. Jin, R. Sukthankar, and Y. Liu, "An efficient algorithm for local distance metric learning," in Proc. Assoc. Advancement Artif. Intell, 2006, pp. 543-548.

[8] B. S. Manjunath and W.-Y. Ma, "Texture features for browsing and retrieval of image data," IEEE Trans. Pattern Anal. Mach. Intell., vol. 18, no. 8, pp. 837-842, Aug. 1996.

[9] A. W. M. Smeulders, M. Worring, S. Santini, A. Gupta, and R. Jain, "Content-based image retrieval at the end of the early years," IEEE Trans. Pattern Anal. Mach. Intell., vol. 22, no. 12, pp. 1349-1380, Dec. 2000.

[10] Pengcheng Wu, Steven C. H. Hoi, Peilin Zhao, Chunyan Miao, and Zhi-Yong Liu, "Online Multi-Modal Distance Metric Learning with Application to Image Retrieval", IEEE TRANSACTIONS ON KNOWLEDGE AND DATA ENGINEERING, VOL. 28, NO. 2, FEBRUARY 2016

[11] Y. Rubner, C.Tomasi, and L. J. Guibas, "The earth movers distance as a metric for image retrieval," Int. J. Comput. Vis., vol. 40, p. 2000, 2000. 